\title{
Solute transport in heterogeneous sediments: experimental and theoretical investigations
}

\author{
Ziqi Ma ${ }^{1}$, Zhenxue Dai ${ }^{1}$, Xiaoying Zhang ${ }^{1}$, Chuanjun Zhan ${ }^{1}$, Huili Gong ${ }^{2}$, Lin Zhu ${ }^{2}$, Corey \\ D Wallace ${ }^{3}$, and Mohamad Reza Soltanian ${ }^{3}$ \\ ${ }^{1}$ Jilin University \\ ${ }^{2}$ Capital Normal University \\ ${ }^{3}$ University of Cincinnati
}

July 7, 2020

\begin{abstract}
Abstract: Heterogeneity is crucial for predication of flow and contaminant transport in subsurface formations. To characterize the heterogeneous architecture, the relationship between multimodal correlation of hydraulic conductivity (K) and plume dispersion is investigated through integration of experimental, theoretical, and numerical simulation approaches. The spatial correlation structure of $\mathrm{K}$ in a heterogeneous sedimentary column is investigated by analyzing the covariance components and transition probability structures. The detailed sedimentary facies data of the column ensures the accuracy of heterogeneous sediment characterization. Lagrangian-based transport models were developed to estimate solute dispersion in non-reactive tracer injection experiments. The results show that the model successively predict the solute transport when the spatial correlation structure is well-defined. Dispersivity estimated by the Lagrangian-based model slightly larger than those obtained from the measurements of tracer experiments. Further, the upscaled dispersivity that derived from transition probability is dominated determined by the cross-transition probability structure, while the contribution of auto-transition terms are quite small. The contribution of the cross-transition terms increases with the increasing contrast in mean permeability between different facies. Numerical modeling results confirm that upscaled dispersivity values well capture solute breakthrough behavior along the heterogeneous sediment column. Keywords: Solute transport; Dispersion; Dispersivity; heterogeneity; porous media; Sedimentary structure; Column experiment; Lagrangian-based model.
\end{abstract}

\section{Hosted file}

main text file.docx available at https://authorea.com/users/340603/articles/467764-solutetransport-in-heterogeneous-sediments-experimental-and-theoretical-investigations 


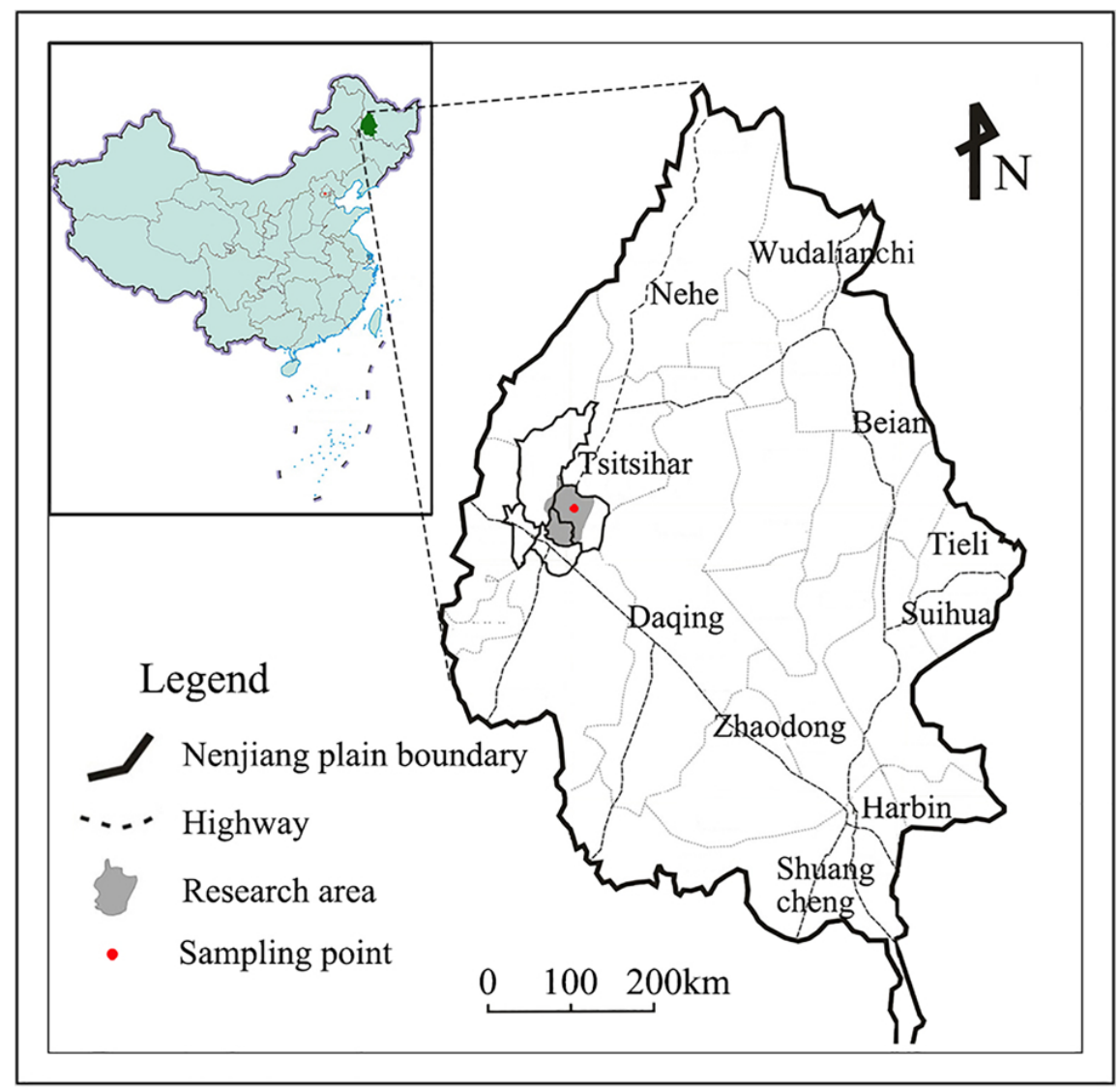

Figure 1. Location of the study area in Tsitsihar, northeast China.

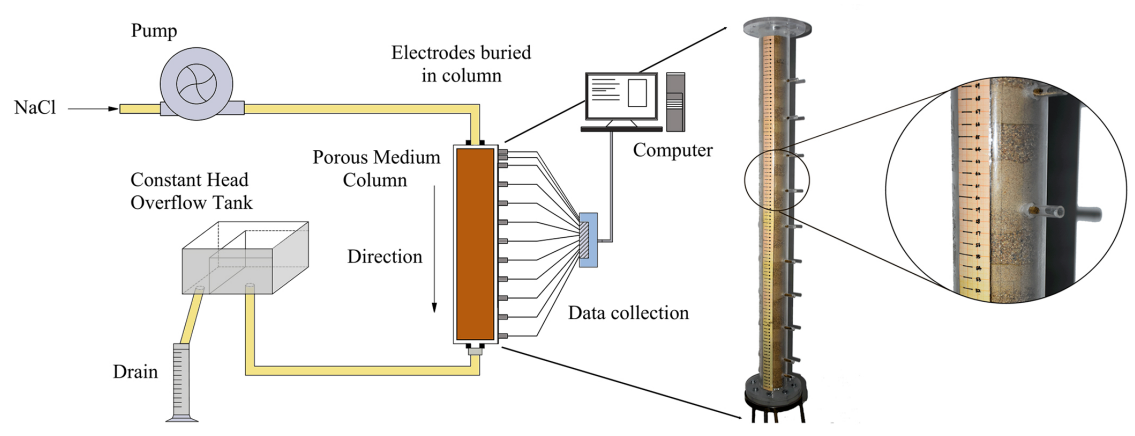

Figure 2. Diagram of the experimental setup and the picture of heterogeneous soil column. 

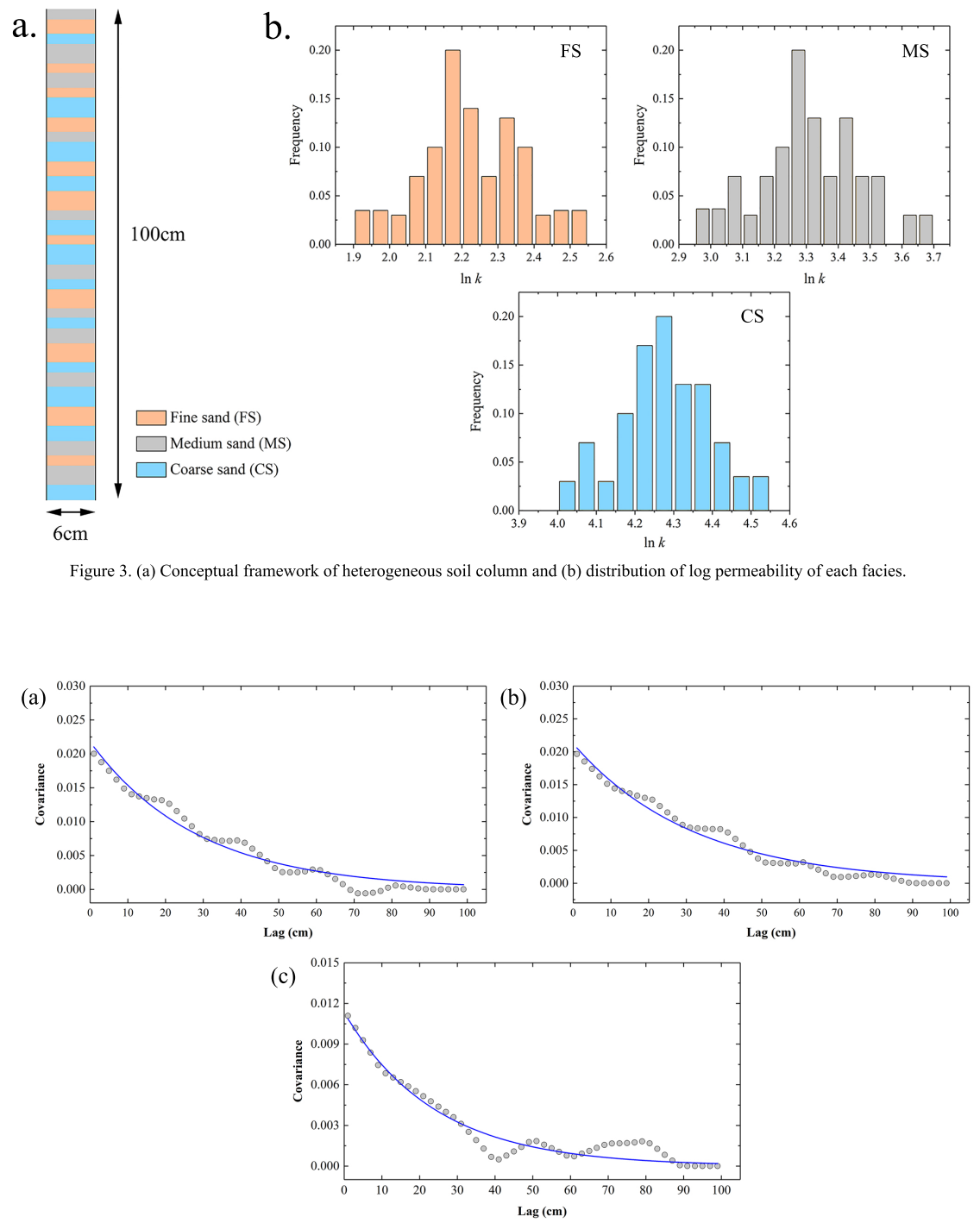

Figure 5. Auto-covariances with sample (gray dots) and fitted model results (solid blue lines). (a), (b) and (c) represent the auto-covariance of fine, medium and coarse sand, respectively. 


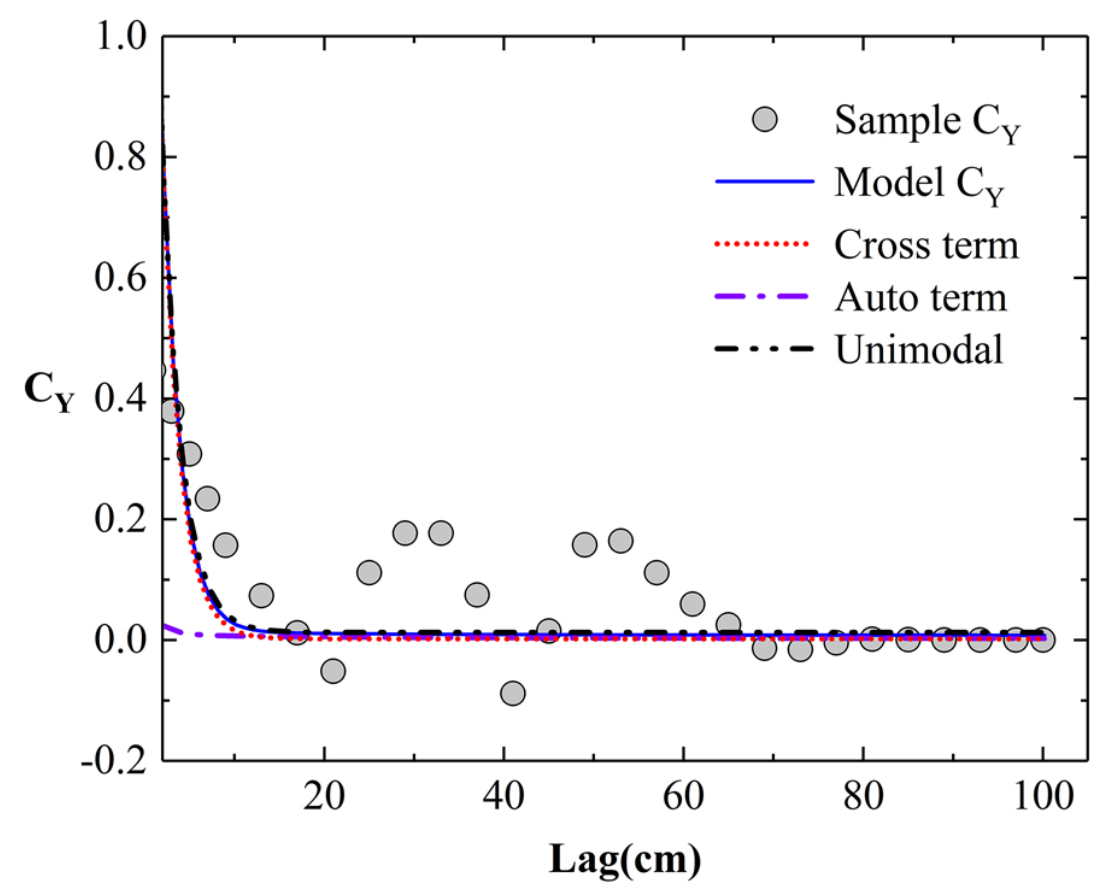

Figure 6. Global covariances with sample (gray dots) and fitted model results (solid blue lines). The contributions of cross- and auto-terms are also plotted. Unimodal covariance is the one calculated value using a single, global integral scale of $2.22 \mathrm{~cm}$. 


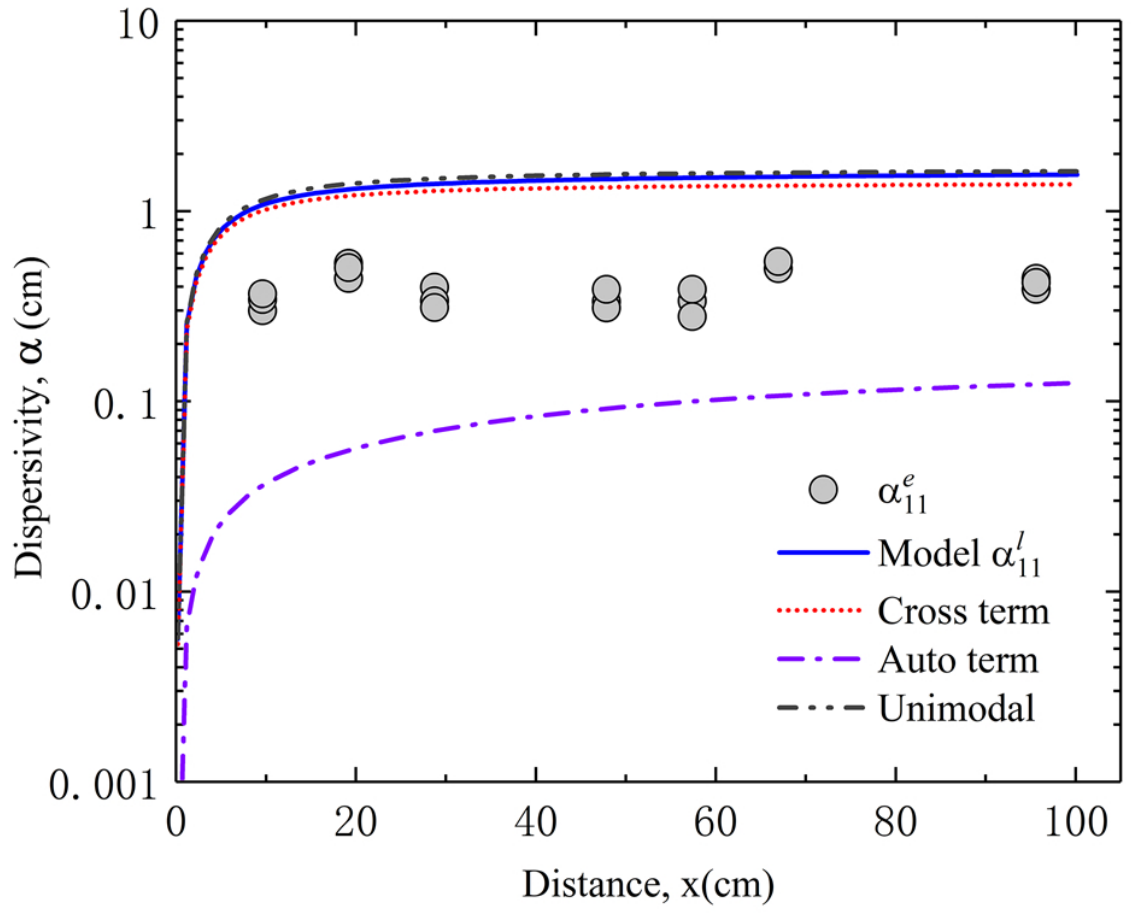

Figure 7. Dispersivities at different distances. Gray dots represent the dispersivities calculated from the experimental data. Solid blue line is the result of the Lagrangian-based model. The contribution of crossand auto-terms are also plotted. Unimodal (upscaled) dispersivity is the one calculated value using a single, global integral scale of $2.22 \mathrm{~cm}$. 
(a)

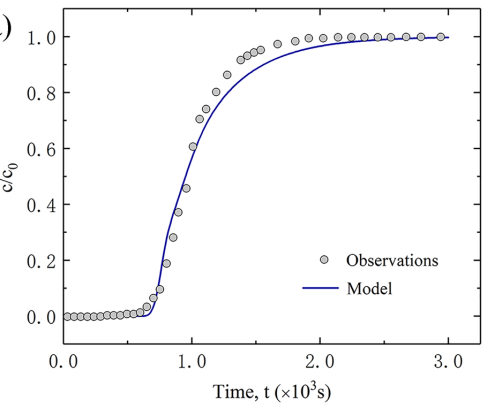

(c)

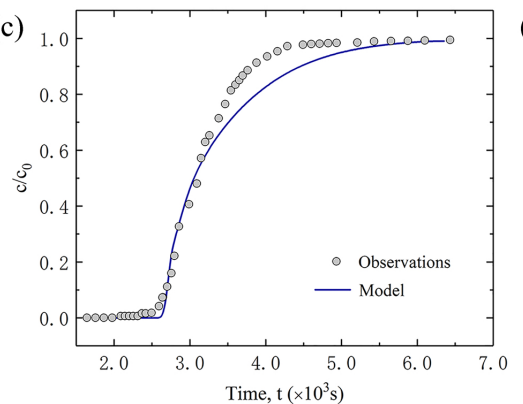

(e)

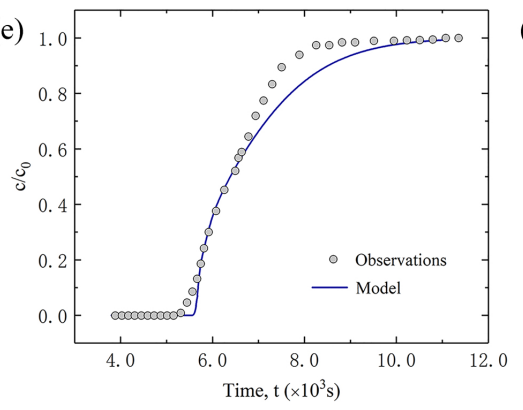

(b)

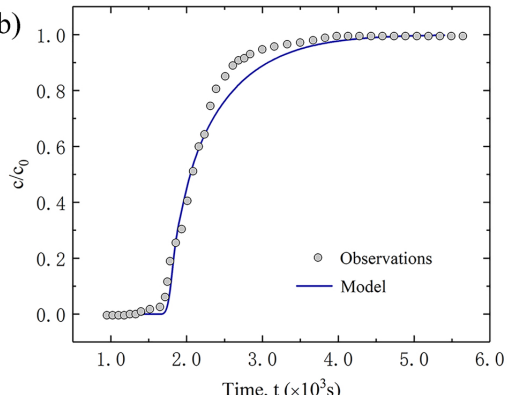

(d)

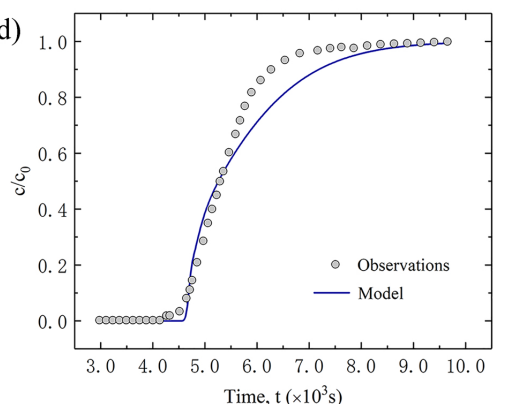

(f)

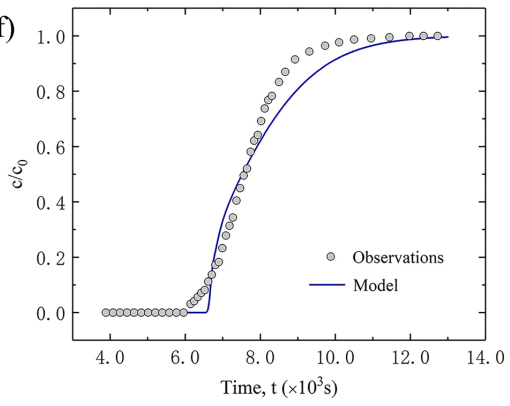

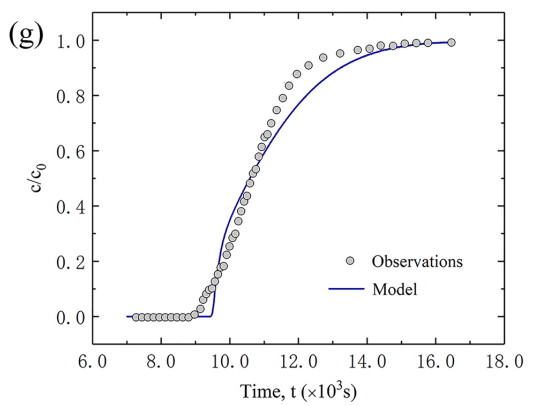

Figure 8. Breakthrough curves at different distances. (a), (b), (c), (d), (e), (f) and (g) represent breakthrough curves at 10, 20, $30,50,60,70$ and $100 \mathrm{~cm}$. Gray dots represent the data measured from tracer experiment. Solid blue lines are the results based on the Lagrangian-based model. 